\title{
A journey of a thousand steps ... Qigong walks for health
}

\begin{abstract}
This article discusses some of the health benefits of walking. In particular it discusses two styles of walking drawn from Chinese martial arts and health practices that have been documented to help alleviate stress and stress related illness. It also discusses the differences Situational Stress and Insipient Stress and provides simple descriptions of these Qigong based walking exercises that may be done anywhere and at any time.
\end{abstract}

Keywords: walking, health promotion, situational stress, insipient stress, Qigong, traditional chinese medicine, self-care
Volume I 3 Issue I - 2020

\author{
Bernie Warren, Candace Hind \\ Drama in Education and Community, University of Windsor, \\ Canada
}

Correspondence: Bernie Warren, Drama in Education and Community, University of Windsor/Owner, Canada, Emailmerv123@uwindsor.ca

\section{Introduction}

Over the last 50years modern life has become faster. Life and job stresses have increased. People now eat larger portions, walk less, and there is an alarming rise in obesity and stress related diseases. This article discusses some of the benefits of walking to health. In particular it looks at two styles of walking drawn from martial arts and health practices that have been documented to help alleviate stress and stress related illness. ${ }^{\mathrm{i}}$

\section{Some of the health benefits of walkingi,iii,iv}

Many health organisations endorse walking as one of the best forms of daily exercise. Research suggests walking helps:

A. Lower the rate of weight gain.

a. Reduce body fat.

B. Strengthen memory.

C. Improve management of conditions such as diabetes, hypertension (high blood pressure), high cholesterol.

D. Increase cardiovascular and pulmonary (heart and lung) fitness.

a. Reduce risk of heart disease and stroke.

E. Reduce joint and muscular pain or stiffness.

F. Build stronger bones and improve balance.

G. Increase muscle strength and endurance.

H. Prevent and/or relieve stress.

Conventional Western wisdom suggests that, to gain the maximum health benefits you should walk for at least 30 minutes briskly, meaning that you can still talk but not sing, at least 3 times a week. While this may be true, the benefits of walking can accrue from simply getting "off the couch" and going for slower and more leisurely strolls.

In addition to the obvious walking practice of simply putting one foot in front of the other, there are many other different styles of walking. ${ }^{\text {viv }}$ Chinese martial arts practice employs multiple ways of stepping and walking.
Underlying these movements is Qigong. While "Qigong" is a modern construct, many of the methods that are used today are derived from age-old Chinese traditions-most notably Taoist \& Buddhist longevity (so called immortality) techniques, meditations and martial arts training exercises. These exercises emphasise the cultivation of internal energy by focussing on breathing patterns, physical posture, and coordination which helps stimulate hormone secretion, immune function, and oxygenation of body cells. All of which help to promote health and counter stress and stress related illnesses.

\section{Intense relaxation and incipient stress}

There was a cartoon in a Canadian Medical Journal of a patient asking the doctor how to relax. The doctor dutifully described various ways of relaxing-meditation, mindfulness exercises, Tai Chi, Yoga, Qigong, and so on. To which the patient replied that these methods were too slow.

"I'm learning how to relax, doctor-but I want to relax, harder and faster! I want to be on the cutting edge of relaxation" ... and there you have it! One of the major dichotomies of modern civilization: individuals want to relax, but they can only try to squeeze short amounts of time for relaxation into their busy schedules.

Stressful situations and stressors are everywhere. The inner conflict between our understanding that we need to slow down and pursue a healthy lifestyle, set against the backdrop of the speeds and pressures of modern life, contributes in large part to both Situational Stress and Incipient Stress.

Situational stress tends to happen to us. There are a multitude of events that possess the potential to create Situational Stress e.g. when we are driving, and someone cuts us off or we get in an accident; a person we love gets sick or dies; we are fired from our job.

To a large extent we can only react to Situational Stress. To deal with this, we can employ simple techniques to calm our breathing, slow our heartbeat and pulse rate. To echo the Serenity Prayer, we must accept the things in the situation we cannot change and focus moment by moment on regaining control of those things we can. This is an area where practices such as Tai Chi, Qigong, Yoga, and mindfulness may help. 
On the other hand, incipient stress is cumulative. It begins early in our lives and can become the sum of all the unresolved issues and Situational stress events that we experience. Moreover, we often are not even aware of it. It is carried around on our backs like invisible clothing. This stress is "... more insidious and more harmful in terms of (its) long-term biological consequences". vii

\section{The way of doing things}

ESTRAGON: "I tell you I wasn't doing anything”. VLADIMIR: "Maybe not, but it is the way of doing it that counts, if you want to go on living.", viii

My friend Steve is a very successful business entrepreneur. For relaxation, he goes dancing with his wife, practices Tai Chi, Qigong and swims. However, he tends to do everything in the same way that he engages in his business, with relentless ferocity and intense drive.

However, this approach is NOT always conducive to relaxation. As he has grown older, he has started to develop various ailments, in large part due to his highly successful and stressful career, youth spent as a competitive martial artist, and several car accidents.

Finally, he realized that he was carrying incipient stress and a level of anxiety that he hadn't previously acknowledged. He realized he was engaging in everything, including his relaxation, in a way that was not good for his health. He made a vow to himself to slow down, pause more often, to reduce the time at each activity, and reduce the intensity of his activity to a slow meditative pace.

By reducing the intensity and duration of his "relaxing" pursuits, he started to focus more on his breathing. In addition, he now goes for more aimless walks and is already noticing a difference in his stress level, his body, and his overall health.

\section{Guo lin qigong - anti-cancer walk}

One example of qigong-based walking practices, that have been documented to help alleviate stress and stress related illness, is the Anti-Cancer Walk developed by Madam Guo Lin.

Guo Lin was one of the most famous female Qigong masters of the 20 th century. However, in 1949 she was diagnosed with cervical cancer. She underwent western medical treatment. She had a hysterectomy, chemotherapy and radiation. She developed further metastatic disease and experienced a total of seven operations within ten years. However, after suffering another major relapse and being told she had only six months to live, she revisited the Qigong her grandfather had taught her as a child. Her subsequent research of ancient writings on Qigong practices helped her to develop her own walking regime. This walking regime came to be known as Guo Lin Qigong or more colloquially as Madam Guo Lin Anti-Cancer Walk. Within six months of practicing this for two hours each day, her cancer went into remission. ${ }^{\text {ix }}$

\section{The Guo Lin Qigong walk has three parts}

a. Breathing Like the Wind. ${ }^{\mathrm{x}}$

b. Bear Shuffling Through the Woods. ${ }^{x i}$

c. Raising the Wet Cloth. ${ }^{\text {xii }}$

This walk is perhaps easier to learn if you practice each of its three parts separately. Bringing all of this together requires patience and coordination.
The great effects of this exercise are achieved through coordination and synchronisation of your movements with your breathing as you walk slowly forward twisting your body while raising your arms to the same side.

Ideally this style of walking is best practiced in the morning outside near water or under trees. But as with any Qigong exercise the benefits can be experienced anywhere and at any time of the day.

This practice is best done for up to 2hours a day, as15-minute sessions of walking with a breaks in between each. However, you can start by taking a few steps, then stop and rest; then another few steps and so on. Gradually, as stamina improves, you can build up the length of time you are able to walk.

A great deal has been written about the effectiveness of Guo Lin Qigong and overall it does appear to be an effective complementary therapeutic approach. In particular, studies have shown it to improve Quality Of Life and to decrease depression and anxiety - all of which are important to the mind body connection which strongly influences how a cancer patient responds to treatment. ${ }^{\text {xii }}$

\section{Benefits of walking in a circle $e^{x i v, x v, x v i}$}

Another example of a health promoting Qigong walk is drawn from Bagua Zhang. ${ }^{\text {xvii }}$ Circle Walking is the quintessential Bagua Zhang exercise. ${ }^{\text {xviii }}$

Circle Walking is a powerful non-aerobic cardiovascular exercise that can be practised regularly anywhere. It can range from being at home, in a relatively small space, by pushing back furniture, to any open space outdoors. It does not matter what you use as the focal point of your circle.

\section{Ba gua circle walking helps}

a. Engage and massage your internal organs-especially the kidneys and bowels.

b. Improve the functioning of your vascular system.

c. Strengthen your muscles.

d. Improve Balance.

e. Reduce stress.

f. Boost the immune system.

g. Eliminate toxins.

h. Increase flexibility.

i. Improve cardiovascular health.

All of which are incredibly important not simply for combatting stress but also in improving overall health and vitality.

\section{A journey of a thousand miles begins with a single step}

There are many techniques which help magnify the beneficial effects of simple walking. These include swinging your arms and linking your breath to the walk e.g. as identified above, and very specific arm postures that influence different bodily processes and organs. ${ }^{\text {xvii }}$

It may seem too good to be true. However, making the decision to 
get off the couch and go for a walk is the first step towards a healthy and potentially longer life. Basic walking is a great start. Integrating Qigong based walking patterns takes this further as this help to prevent illness, improve the supply of nutrients and oxygen in your body, and remove toxins and waste material efficiently. The mindful practice of Qigong based walking takes your focus off conscious distractions and the stressors of everyday life- helping to alleviate Situational stress.

The rhythmic movements and breathing allow your body, without conscious thought, to start to reduce Insipient stress, those every day micro stresses, which like extra layers of clothing, have been weighing you down throughout your life. You can start to make this happen by very slowly walking while focussing on your breathing, in a straight line, in a circle, or simply meandering anywhere and at any time for as long or as short as you desire or is appropriate for your capability. The journey to reduce stress within your life begins with your first step.

\section{Acknowledgments}

I (Bernie Warren) wish to acknowledge and pay homage to all my teachers, especially Master George Ling HU and Sensei O’ Tani for introducing me to the benefits of the way of the circle and to Qigong.

\section{Conflicts of interest}

The authors declare that there are no conflicts of interest.

\section{Funding}

None.

\section{(Endnotes)}

${ }^{\mathrm{i}}$ The title derives from Ba Gua Zhan training where it is said that to begin to understand walking the Circle takes a minimum of 1000 steps.

iihttps://www.realsimple.com/health/fitness-exercise/benefitswalking\#walking-9

iiiGlasper A. Walk this way: improving activity levels. $\mathrm{Br} \mathrm{J}$ Nurs. 2017;26(6):362-363

${ }^{i v}$ bhttps://www.betterhealth.vic.gov.au/health/healthyliving/walking-forgood-health

"Mora Marco J, Benzel R. Way of Walking: Eastern Strategies for Vitality, Longevity, and Peace of Mind. Chicago, Illinois: Contemporary Books; 2000. p. 256.

visheng Ken Yun. Walking Kung. York Beach, USA: Samuel Wiser Inc.

viihttps://drgabormate.com/book/

viiiBeckett S. Waiting for Godot. UK: Faber and Faber; 1956.

ixhttp://www.qigongchinesehealth.com/walking_qigong

${ }^{x}$ Breathing like the Wind - Breathing Technique.

A. Breathe through your nose in the following pattern:

a. TWO Short in Breaths.

b. Long Out Breath.

1. ${ }^{\mathrm{xi} B e a r}$ Shuffles through the Woods - Walking Technique.

\section{Start with right side}
A. Stand with your feet in parallel - shoulder width apart.
B. Breathe In

a. Simultaneously swing your hips and shoulders back to the right.

b. Pick up the toes of your right foot.

C. Breathe Out

D. Drive your sshoulder and hip forward.

a. Allow your foot to slide forward in a straight line.

E. Place your heel down level with the toes of your left foot.

F. i.e. Moving leg should not go past the toes of your supporting leg.

G. NB All Movements Are Generated By The Swinging Of Your Hips Forward And Back.

H. Your shoulder and hip move together.

I. Move same arm as leg forward together,

J. i.e. as right hand moves back right heel lifts off the ground.

a. Allow your arms to swing freely throughout this walk.

\section{Repeat above steps on left side}

A. Continue moving forward by alternating movement of left and right leg.

B. The movement is meant to be slow and rhythmic and

a. Similar to cross country skiing/snowshoeing.

2. xii Raising the Wet Cloth - Twisting the Body Technique.

\section{Part A}

A. Imagine picking up a piece of wet silk cloth or pre-pasted wallpaper.

a. Relax stomach, back straight, using legs for balance.

b. First three fingers almost touch as you lift hands up in front of body to eyebrow height.

c. Keep wrists curved but relaxed as they rise to eyebrow level.

B. At eyebrow level raise your fingers so that your palms are flat $\&$ facing away from you.

C. Slowly move your arms down towards the floor.

a. As though you are mopping wallpaper to a wet glass screen.

D. When your hands reach navel level,

E. Repeat sequence picks up wet cloth.

a. Make sure you exhale as you "mop down"

\section{Part B}

a. Follow directions for Part a BUT.

b. As you rise your arms slowly use your waist to turn your shoulders and hips away from your centre.

c. Your arms stay in front of your body.

d. First to Turn to the Right Side.

e. As you lower your arms TURN your body slowly from the waist back to centre.

f. Repeat on the Left Side.

xiiihttps://books.google.ca/books?hl=en\&lr=\&id=1YnP0Jx-C\&oi=fnd\&pg $=\mathrm{PA} 209 \& \mathrm{dq}=$ Walking + Qigong $+\mathrm{Guo}+$ Lin\&ots $=\mathrm{tAjvukXWZV \& sig=j2HF}$ yYquEcxVG4EBYAauyzhhTY $\# \mathrm{v}=$ onepage $\& \mathrm{q} \& \mathrm{f}=$ fals 
${ }^{\text {xiv } h t t p s: / / p a k u a c h a n g j o u r n a l . c o m / c i r c l e W a l k . p h p ? p a g e ~}=6$

${ }^{x v h t t p s: / / p a k u a c h a n g j o u r n a l . c o m / c i r c l e W a l k . p h p ? p a g e=4 ~}$

xvihttps://www.circlewalking.com/walk-the-circle-london/

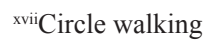
A. Begin by moving Counter-clockwise.
B. Start with your feet together.
C. Turn your hips slightly towards the centre of the circle.
D. Move your Right (Outside) foot forward in a circular motion.
E. Then drive your Left (inside) foot forward in a straight line.
a. Until it is parallel with the Right foot.

F. Turn at the waist slightly as you walk

a. Do Not Place Weight On Pivoting Leg!
G. To avoid strain on your knees - Transfer weight to your turning leg as you move.

a. Sixteen steps usually complete a full circle.

H. To change direction

a. Come to a complete stop with your feet together.

b. Turn your hips slightly towards the centre of the circle.

c. Move your Left foot forward in a circular motion towards the centre of the circle.

d. Then drive your Right foot forward in a straight line.

e. Then move as above.

xviiihttps://pakuachangjournal.com/circleWalk.php?page=5 